\title{
Time-dependent conduction current in lithium niobate crystals with charged domain walls
}

\author{
V. Ya. Shur, ${ }^{1,2, a)}$ I. S. Baturin, ${ }^{1,2}$ A. R. Akhmatkhanov, ${ }^{1,2}$ D. S. Chezganov, ${ }^{1}$ and A. A. Esin ${ }^{1}$ \\ ${ }^{1}$ Ferroelectric Laboratory, Institute of Natural Sciences, Ural Federal University, Ekaterinburg 620000 , \\ Russia \\ ${ }^{2}$ Labfer Ltd., 620014 Ekaterinburg, Russia
}

(Received 1 August 2013; accepted 20 August 2013; published online 6 September 2013)

\begin{abstract}
We present the experimental study of the increase and decrease of the abnormal conduction current appeared during polarization reversal at elevated temperatures $\left(120-250{ }^{\circ} \mathrm{C}\right)$ in stoichiometric and $\mathrm{MgO}$ doped lithium niobate single crystals. It is shown that the conduction current is caused by existence of the through charged domain walls. The time dependence of the conduction current has been measured in low electric field immediately after partial switching. The maximal value of the conduction current in crystal with through charged domain walls is of 4-5 orders of magnitude higher than in initial single domain state. The activation energy is $1.1 \mathrm{eV}$. (C) 2013 AIP Publishing LLC. [http://dx.doi.org/10.1063/1.4820351]
\end{abstract}

The recent interest in the abnormally high electrical conductivity along the ferroelectric domain walls ${ }^{1-6}$ is due to the possible applications of this effect in high-density memory storage and reconfigurable nanoscale electronic circuits. ${ }^{2}$ It is important to point out that the strong increase of the bulk conductivity in the vicinity of the charged domain walls (CDW) has been revealed in seventies in the single crystals of semiconductor-ferroelectric SbSI and has been investigated both theoretically ${ }^{7-10}$ and experimentally. ${ }^{11,12}$ The effect has been attributed to the increase of the free carrier density near CDW. ${ }^{7-10}$ Recently, the effect has been studied intensively both in thin film multiferroics and ferroelectrics ${ }^{1,4}$ and in the bulk single crystals of $\mathrm{BaTiO}_{3}$ (Ref. 6) and $\left.\mathrm{LiNbO}_{3}(\mathrm{LN})\right)^{5,13,14}$ The conduction current $\left(j_{c}\right)$ measurements were made usually by macroscopic electrodes ${ }^{5,11,12}$ and by conductive tip of the atomic force microscope. ${ }^{1,13,15}$

The strong increase of the bulk conductivity during the poling process induced by formation of CDW has been revealed in $\mathrm{MgO}$ doped $\mathrm{LN}(\mathrm{MgOLN}){ }^{5}$ Recently, the strong increase of the conductivity along CDW during illumination of $\mathrm{MgOLN}$ with photoactive light has been demonstrated using high resolution conduction AFM method. ${ }^{13}$

The single crystals of LN family are widely used for nonlinear optical applications. ${ }^{16}$ Moreover, LN crystals can be studied as the model ferroelectrics due to: (1) simple domain structure with only $180^{\circ}$ domain walls, (2) high quality wafers available, (3) ability to apply various nondestructive methods of the domain visualization with sub-micron spatial resolution, ${ }^{17,18}$ and (4) possibility to increase the bulk screening efficiency by heating and doping.

In this paper we present the experimental study of the abnormal increase and decrease of $j_{c}$ appeared during polarization reversal in stoichiometric lithium niobate (SLN) and $\mathrm{MgOLN}$ crystals at elevated temperatures $\left(120-250^{\circ} \mathrm{C}\right)$. The choice of these representatives of LN family is caused by

\footnotetext{
${ }^{\text {a) }}$ Author to whom correspondence should be addressed. Electronic mail: vladimir.shur@usu.ru.
}

their lower coercive field and higher bulk conductivity as compared to the conventional congruent LN.

LN crystals of two different compositions were studied: the commercially available Z-cut wafers of SLN single crystals from SAES Getters (Italy) and congruent LN doped with $5 \mathrm{~mol} . \% \mathrm{MgO}$ from Yamaju Ceramics (Japan). The typical sample dimensions were $1 \times 20 \times 20 \mathrm{~mm}^{3}$. Both polar surfaces of the wafer were polished to optical grade.

The external electric field was applied using evaporated or DC magnetron sputtered 100-nm-thick Cr electrodes. Z-polar surface was covered completely by solid electrode, whereas the array of circular electrodes with diameter $2 \mathrm{~mm}$ and period of $2.5 \mathrm{~mm}$ was deposited on $\mathrm{Z}+$ polar surface.

The scheme of experimental setup for polarization reversal at elevated temperature and subsequent measurement of $j_{c}$ in low field is presented in Fig. 1(a). The sample was fixed by pressure contacts in Teflon bath with silicone oil (Fig. 1(b)). The oil temperature was maintained constant in the range from $100^{\circ} \mathrm{C}$ to $200^{\circ} \mathrm{C}$ using coil connected with solid-state relay and proportional-integral-derivative controller OVEN TPM-151 operating in pulse-width modulation mode with pulse-repetition interval $1 \mathrm{~s}$. The temperature was measured by K-type thermocouple.

The commutation circuit with three relays controlled by NI PCI-6251 multifunctional data acquisition board (designated as "Switch" in Fig. 1(a)) was used for fast reconnection of the sample from polarization switching circuit to $j_{c}$ measurement circuit. The commutation time was about few milliseconds.

The voltage signals for polarization reversal were generated by NI PCI-6251 board and amplified by high-voltage amplifier TREK 20/20C (TREK, Inc., USA). Precision series resistor $(200 \Omega)$ was used for measurement of the current during polarization reversal. The experimental setup allowed us to realize in situ visualization of the domain structure evolution using Carl Zeiss LMA10 polarizing microscope with spatial resolution about few microns.

The circuit for conduction current measurement included the picoamperemeter Keithley 6487 (Keithley Instruments, Inc., USA) controlled by computer using IEEE-488.2 

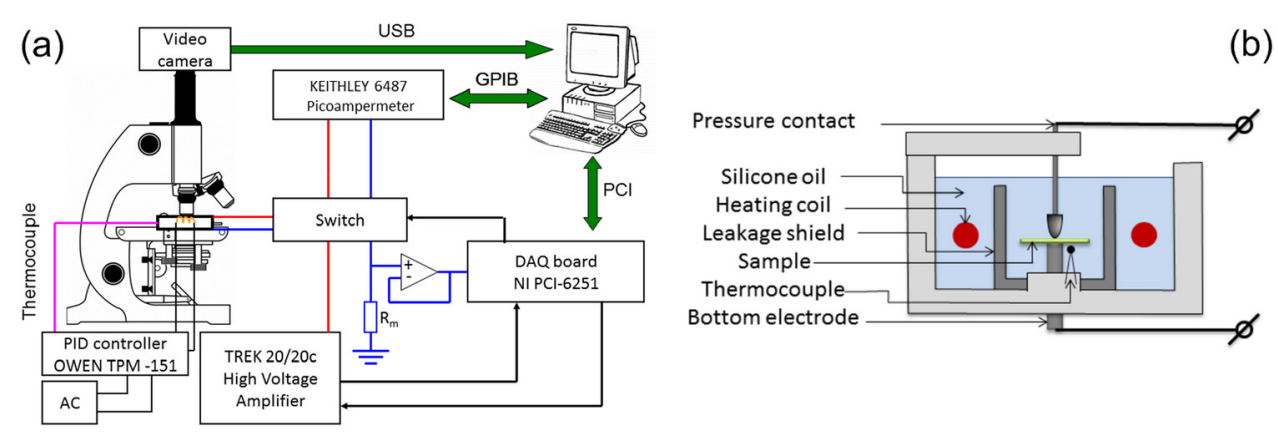

FIG. 1. (a) Experimental setup for polarization reversal and subsequent $j_{c}$ measurement. (b) Teflon bath with the silicone-oil-immersed sample holder.

(General Purpose Interface Bus, GPIB) interface and external signal generator Agilent 33210A (Agilent technologies, USA). The internal analogous low-pass filter of the picoamperemeter allowed us to reduce the noise level of the measured $j_{c}$.

The time dependence of $j_{c}$ was recorded just after partial polarization reversal. Thus, the experimental procedure can be divided in two subsequent stages (Fig. 2).

At the first stage the unipolar rectangular pulse of high field was applied to the single circular electrode on $\mathrm{Z}+$ surface connected to the high voltage amplifier through the switching circuit. The current and the set of instantaneous domain images were in situ recorded during switching. The amplitude and the length of the switching pulse were adjusted to obtain partial polarization reversal of the electrode area. For MgOLN samples the pulse amplitude ranged from 1.5 to $1.8 \mathrm{kV} / \mathrm{mm}$ and for SLN from 2 to $3 \mathrm{kV} / \mathrm{mm}$. The same pulse duration about $5 \mathrm{~s}$ was used for all experiments.

At the beginning of the second stage the sample was commutated to $j_{c}$ measurement circuit (Fig. 2). The low voltage rectangular pulses with amplitude $10 \mathrm{~V}$ (unipolar pulse or series of bipolar rectangular pulses with period ranged from 1 to $10 \mathrm{~s}$ ) were applied.

After partial switching and recoding of $j_{c}$ the electrodes were removed by Chrome Etch $\mathrm{N}^{\mathrm{O}} 1$ (Technic, France). The selective chemical etching in hydrofluoric acid (HF) for 2-5 min at room temperature was used for revealing of the static domain patterns. The surface reliefs corresponding to the domain pattern at both polar surfaces and $\mathrm{Y}$ cross-section were visualized by Olympus BX51 optical microscope using dark field mode (Fig. 3).

The obtained high increase of the current at the end of the switching stage has been attributed to the dominance of

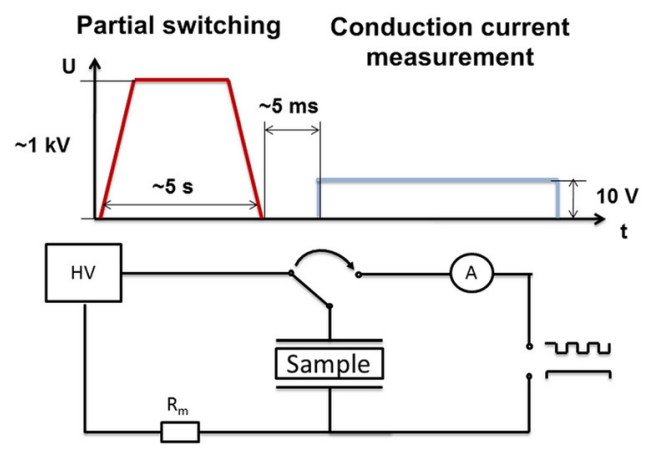

FIG. 2. Waveforms of high-voltage pulse for partial polarization reversal and low-voltage pulse for $j_{c}$ measurement. $j_{c}$ input (Fig. 4). In this case the measured charge was from 10 to 100 times larger than the polarization reversal charge for the switched area covered by electrode. The analysis of the domain images ( $\mathrm{Z}+$ and $\mathrm{Z}$-views, and $\mathrm{Y}$ cross-section) and their comparison with the current data demonstrated that the high $j_{c}$ appeared at the moment, when the through CDW was formed (Fig. 3). Thus, the delay time between the start of the switching process and the appearance of the high $j_{c}$ corresponds to the time, which is necessary for domain growth through the sample.

Increase and subsequent decrease of $j_{c}$ recorded just after partial polarization reversal in SLN have been revealed (Fig. 5(a)). It has been found that the maximal value of $j_{c}$ in multidomain state with through charged domain walls is of 4-5 orders of magnitude higher than in initial single domain state. The time dependences of $j_{c}$ for both processes have been successively fitted by exponential law. The temperature
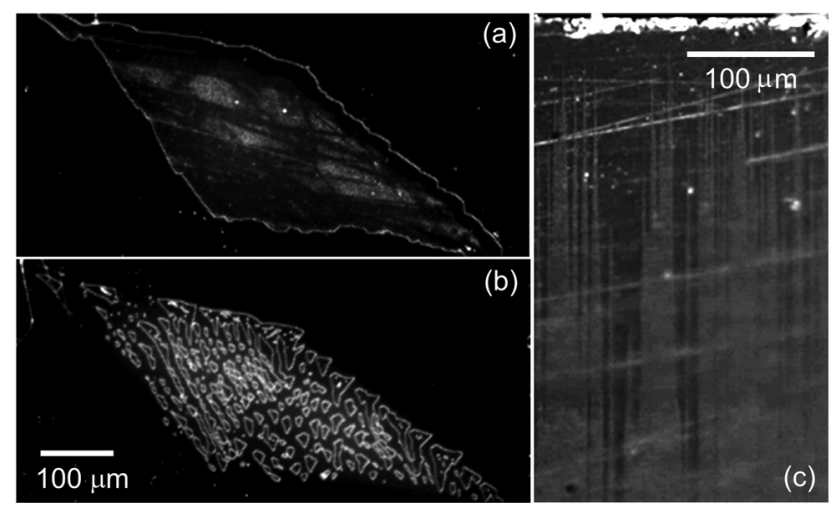

FIG. 3. The domain structure images on polar surfaces (a) $\mathrm{Z}+$, (b) $\mathrm{Z}-$, and (c) on Y-cross section in MgOLN after partial polarization reversal. Optical microscopy in dark field mode after selective chemical etching.

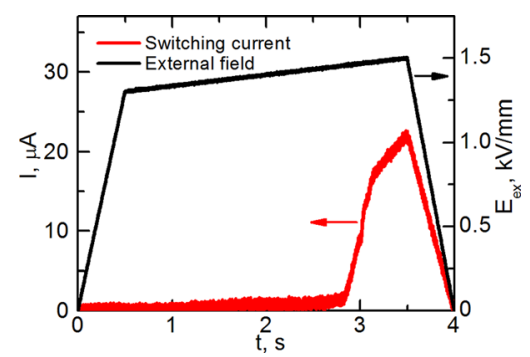

FIG. 4. Waveform of the external field pulse and corresponding switching current in MgOLN. $\mathrm{T}=250^{\circ} \mathrm{C}$. 

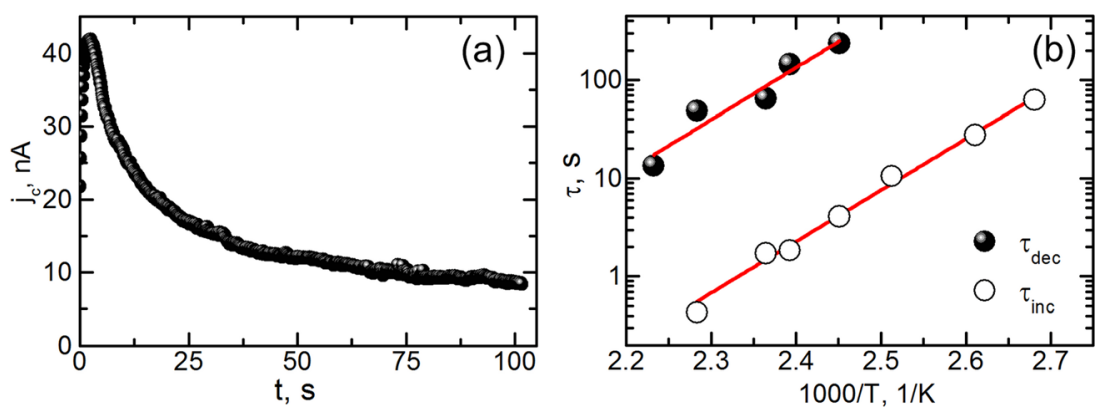

FIG. 5. (a) $j_{c}$ measured just after partial polarization switching $\left(\mathrm{T}=150^{\circ} \mathrm{C}\right)$. (b) Temperature dependence of $j_{c}$ time constants for current increase $\left(\tau_{\text {inc }}\right)$ and current decrease $\left(\tau_{\mathrm{dec}}\right)$ in SLN. Experimental points fitted by Arrhenius law.

dependence of the extracted time constants allowed us to reveal the value of activation energy for both processes equal to $1.1 \pm 0.1 \mathrm{eV}$ (Fig. 5(b)).

The input of $j_{c}$ was studied at different stages of the polarization reversal process. $j_{c}$ maxima were measured in MgOLN during application of the series of $5 \mathrm{~s}$ rectangular pulses with period about $100 \mathrm{~s}$ needed for sufficient decrease of $j_{c}$ (Fig. 6). The observed $j_{c}$ value was negligibly low after the first and the second pulses. The current maximum increased essentially after subsequent four pulses and decreased drastically during further switching (Fig. 6). Negligible $j_{c}$ was observed after the last pulse.

The obtained increase and decrease of the abnormally high $j_{c}$ are due to time variation of the conductivity along the through CDW. The bulk screening of the depolarization field produced by the bound charges existing at the CDW leads to the charge accumulation. The formation of the space charge region results in increase of $j_{c}$ caused by appearance and growth of the "conductive layer" in the bulk. The subsequent decrease of $j_{c}$ can be attributed to the capture of the free charges at the deep traps. ${ }^{19,20}$ The obtained value of the activation energy for both $j_{c}$ increase and decrease can be attributed to conductivity related to lithium vacancies. ${ }^{19,20}$

The increase and decrease of $j_{c}$ maximum during switching by pulse series are caused by increase and decrease of the area of the newly appeared through conductive layers. This behavior is qualitatively similar to the switching current obtained during conventional polarization reversal process.

Conduction current was measured during polarization reversal at elevated temperatures $\left(120-250^{\circ} \mathrm{C}\right)$ in stoichiometric and $\mathrm{MgO}$ doped lithium niobate single crystals. Comparison of $j_{c}$ data and domain images revealed on polar surfaces and Y-cross-section allowed us to reveal that the abnormally high $j_{c}$ appeared at the moment when the through charged domain

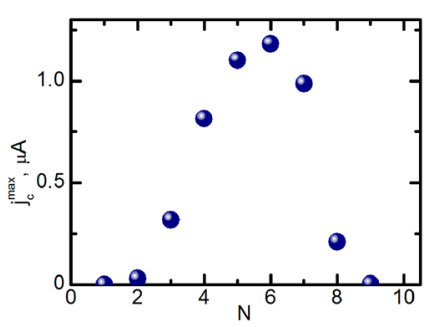

FIG. 6. Dependence of $j_{c}$ maximum on the number of the switching pulses in $\mathrm{MgOLN}$. $\mathrm{T}=150^{\circ} \mathrm{C}$. walls were formed. The time dependence of $j_{c}$ was measured in low field immediately after partial polarization reversal by high electric field. The exponential increase and subsequent exponential decrease of $j_{c}$ were revealed. It was shown that the maximal value of $j_{c}$ in multidomain state with through charged domain walls was of 4-5 orders of magnitude higher than in the initial single domain state. The value of the activation energy $1.1 \mathrm{eV}$ was extracted from the temperature dependence of the time constants for $j_{c}$ increase and decrease. The input of $j_{c}$ was studied during switching by pulse series at different stages of the polarization reversal process. The increase and decrease of $j_{c}$ maximum were caused by increase and decrease of the area of the newly appeared through conductive layers. It was proposed that $j_{c}$ increase was caused by the charge accumulation in the vicinity of the charged domain wall. The subsequent $j_{c}$ decrease was attributed to the capture of the free charges at the deep traps.

The equipment of the Ural Center for Shared Use "Modern Nanotechnology," Institute of Natural Sciences, Ural Federal University was used. The research was made possible in part by RFBR and the Government of Sverdlovsk region (Grant No. 13-02-96041-r-Ural-a), by RFBR (Grant Nos. 11-02-91066-CNRS-a and 13-0201391-a), and with the financial support of young scientists in terms of Ural Federal University development program.

${ }^{1}$ J. Seidel, L. W. Martin, Q. He, Q. Zhan, Y.-H. Chu, A. Rother, M. E. Hawkridge, P. Maksymovych, P. Yu, M. Gajek, N. Balke, S. V. Kalinin, S. Gemming, F. Wang, G. Catalan, J. F. Scott, N. A. Spaldin, J. Orenstein, and R. Ramesh, Nat. Mater. 8, 229 (2009).

${ }^{2}$ J. Seidel, J. Phys. Chem. Lett. 3, 2905 (2012).

${ }^{3}$ E. A. Eliseev, A. N. Morozovska, G. S. Svechnikov, V. Gopalan, and V. Ya. Shur, Phys. Rev. B 83, 235313 (2011).

${ }^{4}$ P. Maksymovych, A. N. Morozovska, P. Yu, E. A. Eliseev, Y.-H. Chu, R. Ramesh, A. P. Baddorf, and S. V. Kalinin, Nano Lett. 12, 209 (2012).

${ }^{5}$ K. Mizuuchi, A. Morikawa, T. Sugita, and K. Yamamoto, J. Appl. Phys. 96, 6585 (2004).

${ }^{6}$ T. Sluka, A. K. Tagantsev, P. Bednyakov, and N. Setter, Nat. Commun. 4, 1808 (2013).

${ }^{7}$ B. M. Vul, G. M. Guro, and I. I. Ivanchik, Ferroelectrics 6, 29 (1973).

${ }^{8}$ B. V. Selyuk, Ferroelectrics 6, 37 (1973).

${ }^{9}$ G. M. Guro, I. I. Ivanchik, and N. F. Kovtonyuk, Sov. Phys. Solid State 10, 100 (1968).

${ }^{10}$ G. M. Guro, I. I. Ivanchik, and N. F. Kovtonyuk, Sov. Phys. Solid State 11, 1574 (1970).

${ }^{11}$ A. A. Adonin and A. A. Grekov, Sov. Phys. Solid State 16, 364 (1974).

${ }^{12}$ A. A. Grekov, A. A. Adonin, and N. P. Protsenko, Ferroelectrics 13, 483 (1976). 
${ }^{13}$ M. Schröder, A. Haußmann, A. Thiessen, E. Soergel, T. Woike, and L. M. Eng, Adv. Funct. Mater. 22, 3936 (2012).

${ }^{14}$ I. S. Baturin, D. S. Chezganov, A. A. Esin, A. R. Akhmatkhanov, and V. Ya. Shur, "Charged domain wall conductivity in lithium niobate and lithium tantalate single crystals at elevated temperatures," Abstract Book of 11th European Meeting on Ferroelectricity (EMF-2011) (The Institute for Solid State Chemistry Bordeaux, 2011), pp. 2C-3O.

${ }^{15}$ S. Farokhipoor and B. Noheda, Phys. Rev. Lett. 107, 127601 (2011).
${ }^{16}$ R. L. Byer, J. Nonlinear Opt. Phys. Mater. 6, 549 (1997).

${ }^{17}$ P. S. Zelenovskiy, M. D. Fontana, V. Y. Shur, P. Bourson, and D. K. Kuznetsov, Appl. Phys. A 99, 741 (2010).

${ }^{18}$ E. Soergel, Appl. Phys. B 81, 729 (2005).

${ }^{19}$ V. M. Fridkin, Ferroelectric Semiconductors (Consultants Bureau, New York, 1980).

${ }^{20}$ A. M. Prokhorov and Y. S. Kuz'minov, Physics and Chemistry of Crystalline Lithium Niobate (Hilger, New York, 1990). 
Applied Physics Letters is copyrighted by the American Institute of Physics (AIP).

Redistribution of journal material is subject to the AIP online journal license and/or AIP copyright. For more information, see http://ojps.aip.org/aplo/aplcr.jsp 